Original Article

\title{
Gastrointestinal Complications after Mitral Valve Surgery
}

Iosif Gulkarov, MD, Susan M Trocciola, MD, Christina C Yokoyama, Leonard N Girardi, MD, Karl K Krieger, MD, O Wayne Isom, MD, and Arash Salemi MD

\begin{abstract}
Introduction: Gastrointestinal (GI) complications are well-recognized risks of open heart surgery. However, open heart surgery comes in different shapes and sizes with widely varying pre-operative, intra-operative and post-operative pathologies. The aim of this study was to examine the etiology and risk factors for GI complications after mitral valve surgery. Methods: A retrospective analysis of 565 patients who underwent mitral valve surgery from 2003-2005 was performed. Prospectively collected data included preoperative risk factors, cardiac status, intra-operative data, postoperative GI complications and mortality. Survival was analyzed using log-rank analysis.

Results: In this study population, 13 patients $(2.3 \%)$ had 16 GI complications after mitral valve surgery resulting in an overall mortality of $0.7 \%$. Complications included GI bleed $(n=9)$, cholecystitis $(n=3)$, perforated diverticulitis $(n=1)$ and ischemic bowel $(n=3)$. By univariate analysis, a history of hypertension, chronic renal insufficiency (CRI), hypercholesterolemia, myocardial infarction, congestive heart failure, cardiogenic shock, emergency valve surgery, coronary artery bypass surgery and preoperative vasopressor use were each associated with an increased incidence of GI complications $(p<0.05)$. No increased incidence was seen in patients with atrial fibrillation. On multivariate analysis adjusted for age, cardiogenic shock (OR 8.1; 95\% CI, 1.9-34.8), CRI (OR 8.1; 95\% CI, 2.2-30.0) and vasopressor use (OR 6.5; 95\% CI, 1.3-31.0) remained significant $(\mathrm{p}<\mathbf{0 . 0 2})$. Mean survival (3.2 vs. 5.4 years) was significantly lower $(p<0.05)$ in those with GI complications.

Conclusions: GI complications after mitral valve surgery are infrequent, with a higher incidence in those with cardiogenic shock, CRI or requiring vasopressors. Pre-operative hemodynamic instability may be a bellwether for potential GI complications and should be of more prominent concern in this cohort of patients.
\end{abstract}

Keywords: mitral valve, complications, cardiopulmonary bypass

$\overline{\text { Department of Cardiothoracic Surgery, New York Presbyterian }}$ Hospital, Weill Cornell Medical College, New York, USA

Received: January 7, 2013; Accepted: April 16, 2013 Corresponding author: Arash Salemi, MD. Department of Cardiothoracic Surgery, New York Presbyterian Hospital, Weill Cornell Medical College, Fourth Floor Greenberg Pavilion M-Wing, 525 East 68th Street M404, New York 10021, USA

Email: ars9001@med.cornell.edu

*This paper was presented at the Scientific Exhibit of the American College of Surgeons 2008 Clinical Congress; San Francisco, California, USA; October 12-16, 2008.

(C)2014 The Editorial Committee of Annals of Thoracic and Cardiovascular Surgery. All rights reserved.

\section{Introduction}

Open heart surgery is associated with a small but well-described risk of gastrointestinal (GI) complications. The incidence of these complications following cardiac surgery is low with most series reporting rates less than $1 \%$. However, mortality in these patients is high, ranging from $11 \%-70 \%$ in various studies. ${ }^{1-8)}$

Multiple published reports have examined GI complications after cardiac surgery in general. This represents a heterogeneous group of pathologies. While all series 
have looked at patients undergoing cardiopulmonary bypass, none have stratified patients according to the type of surgical intervention. Cardiac surgical patients present with significantly different pre-morbid conditions ranging from angina to heart failure or arrhythmias. Technically, they also demand varying degrees of bypass time, exposure of the heart chambers, systemic cooling and circulatory arrest. Subsequent complications and potential visceral sequelae may vary depending on the type of surgical procedure performed.

This study was developed to examine the etiology, risk factors and outcomes of patients with GI complications in patients with mitral valve disorders requiring repair or replacement of their mitral valves as part of their operation. Patients with mitral valve disease often present with arrhythmias and/or congestive heart failure. Intraoperatively, these patients require entry into the heart chambers which exposes them to a risk for air and or particulate embolism. We sought to evaluate the unique GI risks associated with mitral valve surgery.

\section{Methods}

The records of 565 consecutive adult patients who underwent mitral valve surgery (199 isolated mitral valve) using cardiopulmonary bypass at a single institution from 2003-2005 were retrospectively analyzed. Prospectively collected data included preoperative risk factors, cardiac status, intra-operative data, post-operative GI complications and mortality. Data collected were based on standard definitions of patient characteristics, diseases severity and outcomes described by the Society for Thoracic Surgery adult cardiac surgery database. The study was reviewed and approved by the Institutional Review Board of the Weill Cornell Medical College.

All patients underwent a median sternotomy with use of $400 \mathrm{IU}$ of heparin $/ \mathrm{kg}$ of body weight for bypass to achieve an activated clotting time (ACT) $>450$ seconds. All valve operations were performed under cardioplegic arrest using cold blood potassium cardioplegia. Moderate perfusion hypothermia $\left(25^{\circ} \mathrm{C}-32^{\circ} \mathrm{C}\right)$ was utilized while on cardiopulmonary bypass. All patients received prophylactic antibiotics and had a nasogastric tube (NGT) placed intra-operatively. The NGT remained in place for a minimum of 12 hours, and longer if the patient remained intubated. Postoperatively, all patients received perioperative GI prophylaxis with proton pump inhibitors. Also, all patients received post-operative therapeutic dose anticoagulation for their mitral surgery.
Patients with GI complications that arose within 30 days of their cardiac procedure were considered for this study. Postoperative GI complications were documented by clinical status and appropriate hematological, biochemical, radiographic and endoscopic exams. Significant GI complications were defined as: GI bleeding- melena or hematemesis associated with a hematocrit drop of at least 2 points; acute cholecystitis- abdominal pain associated with an ultrasound exam demonstrating a thickened gallbladder wall; ischemic bowel-diagnosis based on both clinical (abdominal pain) and laboratory (elevated white blood cell count or lactate) evaluation; perforated viscus-abdominal pain associated with free air on a radiographic study.

Data were analyzed using standard statistical methods. Univariate testing was done with Fischer's exact test for analysis of dichotomous data and Student t-test for continuous data analysis. Continuous data was expressed as a mean \pm standard deviation. Significance was assumed atp $\leq 0.05$. A multivariate logistic regression model with odds ratio (OR) and $95 \%$ confidence intervals (CI) was developed from the results of the univariate analysis, with inclusion variables having a value $p \leq 0.05$. Survival was analyzed using log-rank analysis. Calculations were performed with SPSS (version 11) for windows (SPSS Inc., Chicago, Illinois, USA).

\section{Results}

\section{Demographics}

In this study population, 13 patients $(13 / 565,2.3 \%)$ had 16 GI complications after mitral surgery. GI complications included perforated diverticulitis $(n=1)$, ischemic bowel $(n=3)$, GI bleed $(n=9)$ and acute cholecystitis $(n=3)$. The three patients with acute cholecystitis also had GI bleeding. Five of the thirteen patients with GI complications died prior to discharge for an overall mortality of $38 \%$.

The mean age of patients undergoing mitral valve surgery was $66 \pm 13$ years (range 20-91) and was not significantly different in those with GI complications (Table 1). While the majority of patients 336 (59\%) were men, slightly more women $(n=8,61 \%)$ had GI complications (Table 1, $\mathrm{p}=\mathrm{NS}$ ).

\section{Natural history (Table 2)}

The patient with perforated diverticulitis presented in cardiogenic shock after an acute myocardial infarction (MI) and underwent an emergency mitral valve replacement/coronary artery bypass graft (MVR/CABG) with a 
Table 1 Univariate analysis of patients without GI complications versus with GI complications after mitral valve surgery



GI: gastrointestinal; $C A D$ : coronary artery disease; CVA: cerebral vascular accident; COPD: chronic obstructive pulmonary disease; CRI: chronic renal insufficiency; IABP: intra-aortic balloon pump; NS: not significant

Table 2 Management and outcome of patients with gastrointestinal complications ( $\mathrm{n}=13$ patients $)$

\begin{tabular}{lccc}
\hline Gastrointestinal complication & Medical treatment & Surgical treatment & Mortality \\
\hline Diverticulitis $(\mathrm{n}=1)$ & 1 & 0 & $0 / 1$ \\
Ischemic bowel $(\mathrm{n}=3)$ & 0 & 3 & $2 / 3$ \\
Isolated GI bleed $(\mathrm{n}=6)$ & 6 & 0 & $1 / 6$ \\
GI bleed + acute cholecystitis $(\mathrm{n}=3)$ & 3 & 1 & $2 / 3$ \\
\hline
\end{tabular}

GI: gastrointestinal

perioperative intra-aortic balloon pump (IABP). On post-operative day eight, the patient developed acute abdominal pain and was found to have perforated diverticulitis. She was treated conservatively with intravenous antibiotics and discharged home two weeks later.

There were three patients who developed ischemic colitis after mitral valve surgery, all requiring surgical exploration. Two of these patients underwent emergency cardiac surgery; one with acute mitral regurgitation (MR) after an MI and the second one with an acute MI/ pulmonary edema who arrested prior to sternotomy incision. One patient had abdominal pain and a rising lactate on post-operative day 3 (s/p MVR for acute MR) and on exploration was found to have diffusely necrotic bowel, expiring the next day from worsening acidosis and low flow state. A second patient developed a severe acidosis post-operative day 9 (s/p CABG/MVR with intraoperative arrest) while on high dose vasopressors and was found to have diffusely ischemic small bowel. This patient failed conservative medical management expiring 10 days later from worsening acidosis and low flow 
state. The third patient underwent an elective MVR/ AVR/CABG (AVR: aortic valve replacement) and who developed abdominal pain approximately one month after initial surgery during a prolonged intensive care unit (ICU) stay. Intraoperatively patient was found to have both small and large bowel that was ischemic, but viable and he was successfully treated with medical management and discharged one month after abdominal exploration.

There were six patients who had isolated GI bleeds after mitral valve surgery. None of these patients underwent cardiac surgery as an emergency. All six patients underwent esophagogastroduodenoscopy (EGD), with diffuse gastritis found in five patients and a healed gastric ulcer found in the sixth patient. The one mortality was in the setting of multi-system organ failure from overwhelming sepsis secondary to sternal wound infection and was not attributed to the upper GI bleed.

Three patients had both a GI bleed and acute acalculous cholecystitis. One patient underwent urgent reoperative mitral valve replacement (previous AVR/CABG) secondary to severe pulmonary edema/congestive heart failure (CHF) who had an upper gastrointestinal (UGI) bleed post-operative day 2 secondary to antral erosions from a nasogastric (NG) tube. Approximately one month after surgery during a prolonged ICU stay, the patient developed right upper quadrant pain and was found by ultrasound to have acute cholecystitis. This was initially treated with a cholecystostomy tube and subsequently underwent an open cholecystectomy the next day because the patient did not initially improve. This patient was discharged 10 days later. A second patient underwent an elective reoperative MVR/AVR/CABG (history of open mitral commissurotomies) who developed jaundice and rising liver function tests postoperative day 10 . He was found to have acute cholecystitis that was treated with a cholecystostomy tube. One month after cardiac surgery the patient was found to have a GI bleed and with diagnosed with cytomegalovirus (CMV) colitis via colonoscopy. Three months after initial surgery the patient expired from multi-system organ failure from overwhelming sepsis. A third patient underwent a MVR/ AVR/CABG in the setting of cardiogenic shock and was found to have a bleeding duodenal ulcer on post-operative day 14 that was treated with cauterization and injection via EGD. Six weeks postoperatively the patient developed abdomen pain and an increasing white blood count and was found to have acalculous cholecystitis that was treated with a percutaneous cholecystostomy
Table 3 Multivariate analysis of risk factors predictive of the development of GI complications after mitral valve surgery

\begin{tabular}{lcc}
\hline \multirow{2}{*}{ Variable } & P value & Odds ratio \\
\cline { 3 - 3 } & & $(95 \% \mathrm{CI})$ \\
\hline Chronic renal insufficiency & 0.002 & $8.1(2.2-30.0)$ \\
Preop cardiogenic shock & 0.005 & $8.1(1.9-34.8)$ \\
Preop vasopressors & 0.021 & $6.5(1.3-31.0)$ \\
\hline GI: gastrointestinal; CI: confidence interval &
\end{tabular}

tube. This patient died the following day from cholangitis related sepsis.

\section{Risk factors}

Univariate analysis was used to compare preoperative and intra-operative co-morbidities in those with and without GI complications after mitral valve surgery (Table 1). A history of hypertension, hypercholesterolemia, myocardial infarction, cardiac angioplasty, concomitant coronary artery bypass surgery, congestive heart failure and chronic renal insufficiency (CRI) were each associated with a significantly increased risk $(\mathrm{p}<.05)$ of GI complications. A history of diabetes, stroke, smoking and chronic obstructive pulmonary disease (COPD) did not predict a significantly higher risk of GI complications ( $\mathrm{p}=\mathrm{NS}$ ) (NS: not significant).

The condition of the patient immediately preoperatively predicted the risk of GI complications. GI complications were more likely to occur in those that were preoperatively in cardiogenic shock (39\% vs. $1.5 \%, \mathrm{p}<.001)$ or unstable requiring pre-operative vasopressors (39\% vs. $5 \%$, $\mathrm{p}<.001$ ) or an intra-aortic balloon pump (39\% vs. $12 \%$, $\mathrm{p}<.02$ ). Patients with GI complication did not have significantly higher rates of preoperative atrial fibrillation compared with those who did not have GI complications ( $62 \%$ vs. $41 \%, \mathrm{p}=\mathrm{NS})$.

Intra-operative data demonstrated minimal differences between the two groups. The cross clamp time $(98 \pm 28$ min vs. $80 \pm 30 \mathrm{~min}, \mathrm{p}=\mathrm{NS})$ and bypass time $(138 \pm 60$ min vs. $114 \pm 48 \mathrm{~min}, \mathrm{p}=\mathrm{NS}$ ) were not significantly longer for those with GI complications compared to those without GI complications. Similar number of patients in each cohort had isolated valve surgery (GI complication: $36 \%$ vs. no complication: $30 \%, \mathrm{p}=\mathrm{NS}$ ). However, patients with GI complications were significantly more likely to undergo concomitant coronary artery bypass surgery $(\mathrm{N}=9,69 \%)$ and placement of an intraaortic balloon pump by the end of the cardiac procedure 
Table 4 Survival data comparing patients without GI complications versus with GI complications after mitral valve

\begin{tabular}{lccc}
\hline & No GI complication & GI complication & \multirow{2}{*}{ P value } \\
\cline { 2 - 3 } & $\mathrm{n}=552$ & $\mathrm{n}=13$ & \\
\hline Length of stay & 9.7 days & 40 days & $\mathrm{P}<0.001$ \\
30 day survival & $533 / 552(97 \%)$ & $11 / 13(85 \%)$ & $\mathrm{NS}$ \\
Mean survival & 5.4 years & 3.2 years & $\mathrm{P}<0.05$ \\
\hline
\end{tabular}

GI: gastrointestinal; NS: not significant

( $\mathrm{n}=5,39 \%)$ compared with those that did not have a GI complication $(\mathrm{N}=167 ; 30 \%, \mathrm{p}<0.005 ; \mathrm{N}=66,12 \%$, $\mathrm{P}<.02)$.

Variables that were found to be significant were entered into a multivariate model to determine which factors were the strongest predictors of GI complications after mitral valve surgery (Table 3). On multivariate analysis adjusted for age, cardiogenic shock (OR 8.1; 95\% CI, 1.9-34.8) (OR: odds ratio; CI: confidence interval), CRI (OR 8.1; 95\% CI, 2.2-30.0) and vasopressor use (OR 6.5; 95\% CI, 1.3-31.0) remained significant (p $<.02)$.

Both the length of stay and survival were significantly different between the two groups (Table 4). Patients with GI complications had a longer length of stay (40 days vs. 9.7 days, $\mathrm{p}<0.001$ ) and a shorter mean survival (3.2 years vs. 5.4 years, $\mathrm{p}<.05)$. In the patients with GI complications, 2/13 (15\%) died within 30 days of their initial procedure.

\section{Discussion}

Previous studies have found that GI complications after cardiac surgery are relatively rare with an incidence ranging from $0.3 \%$ to $2 \%$, and a high mortality rate of $11 \%$ to $59 \% .^{1-8)}$ In our study, the incidence of GI complications in patients undergoing mitral valve surgery was $2.3 \%$ with a 30-day mortality of $39 \%$, both of which consistent with existing reports. Valvular surgery was suggested to correlate with higher incidence of GI complications both by univariate ${ }^{9-13)}$ and multivariate ${ }^{3,14)}$ analysis, and our incidence is in agreement with this observation. Despite improvements in perioperative care, monitoring, anesthesia, and operative technique, incidence and mortality of GI complications had not changed through the years. These improvements have been offset by an actual change in the cardiac surgery referral pattern, which now includes older and sicker surgical candidates.
At the same time, independent determinants for GI complications have not been extensively studied.

Numerous studies looked at different risk factors trying to elucidate the preoperative, intraoperative and postoperative factors that would correlate with GI complications after cardiac surgery. Only 8 studies thus far used multivariate analysis to identify the independent determinants of GI complications in cardiac surgery. ${ }^{3,7,14-19)}$ Although, the pathogenesis of GI lesions is complex and multifactorial, the major factor implicated in cardiac surgery is reduced systemic blood flow which is affected by multiple perioperative variables.

Relationship between duration of cardiopulmonary bypass and incidence of GI complications is not uniform. There are studies that favor that relationship, ${ }^{14,16,18)}$ and some that do not find a significant relationship. ${ }^{15,17,19)}$ In our analysis, although cardiopulmonary bypass (CPB) time and crossclamp time were higher in the GI complications group, they were not statistically significant both in univariate and multivariate analysis.

Perioperative factors such as cardiogenic shock and vasopressor requirements are good indicators of a low output state. Statistically significant relationship was well demonstrated in our study where cardiogenic shock and vasopressor requirements were correlated with the incidence of GI complications after mitral valve surgery by multivariate analysis. Low flow state often requires placement of IABP which has been identified as a determining factor for abdominal complications in both multivariate ${ }^{7,14,17)}$ and univariate studies. ${ }^{2,3,6,8,10,13,20)}$ In our analysis, although postoperative IABP use was significantly more common in the GI complications group by univariate analysis (Table 1), its impact after logistical regression analysis was not significant.

Concomitant $\mathrm{CABG}$ in addition to mitral valve surgery was also reported to be predictive of GI complications by multivariate analysis by Mohavedi and his coauthors, ${ }^{14)}$ who explained this correlation by longer bypass time 
required for CABG. Yet, in our study bypass time was not significantly different between groups with and without GI complications. Relationship between CABG and GI complications held true in our univariate analysis too (Table 1) and in some other studies (3). It is possible that the presence of coronary artery disease (CAD) that requires concomitant surgical revascularization is associated with vasculopathy in mesenteric bed, thus potentially predisposing a patient to more ischemia in perioperative period.

The relationship between chronic renal failure (CRF), acute renal failure (ARF) and GI complications after cardiac surgery has been studied in several univariate ${ }^{20,21)}$ and multivariate ${ }^{3,14)}$ analysis publications. In our experience CRF is an independent determinant for GI complications.

GI hemorrhage is the most common abdominal complication following cardiac surgery. ${ }^{6,8,10,20)}$ Valve surgery that requires anticoagulation often increases bleeding. In our series, surgical intervention was not required for isolated GI bleeding; surgery was required only when there was an additional GI complication (ischemic bowel or acute cholecystitis). Majority of patients with GI bleeding can be treated conservatively and rarely require surgery.

The second most common abdominal complication following cardiac surgery is acute mesenteric ischemia (AMI). Incidence of acute mesenteric ischemia after cardiopulmonary bypass is $0.06 \%-0.2 \%$, with relative incidence of $11 \%-27 \%$ in patients that are affected by intraabdominal complications. ${ }^{10,13,22)}$ AMI carries very high mortality $(85 \%-94 \%) .^{10,13,23)}$ In our series, two out of three patients expired following diagnosis of AMI, which suggests that despite significant advances in cardiac critical care AMI continues to remain a very lethal complication. This high mortality is partly due to difficulty of early diagnosis in cardiac surgical patients. Identification of the risk factors associated with GI complications/ mesenteric ischemia should allow for early diagnosis and hence better patient outcomes.

Although, some thrombotic conditions such as cardiac arrhythmias, left ventricular aneurysm, or recent myocardial infarction were reported as a risk factor for mesenteric artery embolism, ${ }^{24)}$ in our study despite the presence of specific anatomic and physiologic conditions associated with mitral regurgitation, namely left atrial enlargement and atrial fibrillation, did not parse out as predictors of GI complications. That confirms the pathophysiology of AMI in patients that underwent cardiac surgery is frequently related to nonocclusive mesenteric arterial ischemia, rather than embolic disease. Decreased systemic blood flow both before and during open heart surgery underlie these complications. Conditions that relate to hypoperfusion have been reported in multiple studies as the risk factors for AMI, and the results of our study appear to be in agreement with that. Known risk factors for AMI are emergent nature of a procedure, ${ }^{22}$ IABP, ${ }^{14,22)}$ failed angioplasty requiring surgery, ${ }^{22)}$ prolonged pump time and advanced age, ${ }^{3,22)}$ reoperative cardiac surgery, ${ }^{23)}$ longer cross-clamp time ${ }^{3,4)}$ and greater inotropic support. ${ }^{4)}$

The third most common GI complication after cardiac surgery $^{6,19,20)}$ is cholecystitis. Leitman and co-workers ${ }^{10)}$ reported 6 cases of acute calculous cholecystitis with a mortality of $73 \%$. The reported mortality from cholecystitis in these patients ranges from $0 \%$ to $73 \% .^{3,10,20)}$ None of the patients in our study had isolated cholecystitis, and one out of three required surgery with overall mortality of $66 \%$. In many of the early published series, acalculous cholecystitis is more prevalent ${ }^{10,25-27)}$ just like it is in our study group, although there are many reports where there is a mixed presence of calculous and acalculous disease. ${ }^{6,8,20)}$

In conclusion, GI complications following isolated or concomitant mitral valve surgery are rare but lethal complications requiring high index of suspicion for GI complications should be considered in patient with conditions of hypoperfusion.

\section{Disclosure Statement}

None of the authors have conflict of interest.

\section{References}

1) Andersson B, Nilsson J, Brandt P, et al. Gastrointestinal complications after cardiac surgery. Br J Surg 2005; 92: 326-33.

2) Bolcal C, Iyem H, Sargin M, et al. Gastrointestinal complications after cardiopulmonary bypass: sixteen years of experience. Can J Gastroenterol 2005; 19: 613-7.

3) D'Ancona G, Baillot R, Poirier B, et al. Determinants of gastrointestinal complications in cardiac surgery. Tex Heart Inst J 2003; 30: 280-5.

4) Garofalo M, Borioni R, Nardi P, et al. Early diagnosis of acute mesenteric ischemia after cardiopulmonary bypass. J Cardiovasc Surg (Torino) 2002; 43: 455-9.

5) Kurt M, Litmathe J, Roehrborn A, et al. Abdominal complications following open-heart surgery: a report of 12 cases and review of the literature. Acta Cardiol 
2006; 61: 301-6.

6) Lazar HL, Hudson H, McCann J, et al. Gastrointestinal complications following cardiac surgery. Cardiovasc Surg 1995; 3: 341-4.

7) Mangi AA, Christison-Lagay ER, Torchiana DF, et al. Gastrointestinal complications in patients undergoing heart operation: an analysis of 8709 consecutive cardiac surgical patients. Ann Surg 2005; 241: 895-901; discussion 901-4.

8) Yilmaz AT, Arslan M, Demirkilc U, et al. Gastrointestinal complications after cardiac surgery. Eur J Cardiothorac Surg 1996; 10: 763-7.

9) Krasna MJ, Flancbaum L, Trooskin SJ, et al. Gastrointestinal complications after cardiac surgery. Surgery 1988; 104: 773-80.

10) Leitman IM, Paull DE, Barie PS, et al. Intra-abdominal complications of cardiopulmonary bypass operations. Surg Gynecol Obstet 1987; 165: 251-4.

11) Moneta GL, Misbach GA, Ivey TD. Hypoperfusion as a possible factor in the development of gastrointestinal complications after cardiac surgery. Am J Surg 1985; 149: 648-50.

12) Simic O, Strathausen S, Hess W, et al. Incidence and prognosis of abdominal complications after cardiopulmonary bypass. Cardiovasc Surg 1999; 7: 419-24.

13) Tsiotos GG, Mullany CJ, Zietlow S, et al. Abdominal complications following cardiac surgery. Am J Surg 1994; 167: 553-7.

14) Movahedi N, Karimi A, Ahmadi H, et al. Laparatomy due to gastrointestinal complications after open heart surgery. J Cardiovasc Surg (Torino) 2011; 52: 111-6.

15) Christenson JT, Schmuziger M, Maurice J, et al. Gastrointestinal complications after coronary artery bypass grafting. J Thorac Cardiovasc Surg 1994; 108: 899-906.

16) Perugini RA, Orr RK, Porter D, et al. Gastrointestinal complications following cardiac surgery. An analysis of 1477 cardiac surgery patients. Arch Surg 1997;
132: $352-7$.

17) Spotnitz WD, Sanders RP, Hanks JB, et al. General surgical complications can be predicted after cardiopulmonary bypass. Ann Surg 1995; 221: 489-96; discussion 496-7.

18) Zacharias A, Schwann TA, Parenteau GL, et al. Predictors of gastrointestinal complications in cardiac surgery. Tex Heart Inst J 2000; 27: 93-9.

19) Vassiliou I, Papadakis E, Arkadopoulos N, et al. Gastrointestinal emergencies in cardiac surgery. A retrospective analysis of 3,724 consecutive patients from a single center. Cardiology 2008; 111: 94-101.

20) Ohri SK, Desai JB, Gaer JA, et al. Intraabdominal complications after cardiopulmonary bypass. Ann Thorac Surg 1991; 52: 826-31.

21) Huddy SP, Joyce WP, Pepper JR. Gastrointestinal complications in 4473 patients who underwent cardiopulmonary bypass surgery. Br J Surg 1991; 78: 293-6.

22) Allen KB, Salam AA, Lumsden AB. Acute mesenteric ischemia after cardiopulmonary bypass. J Vasc Surg 1992; 16: 391-6; discussion 395-6.

23) Klempnauer J, Grothues F, Bektas H, et al. Acute mesenteric ischemia following cardiac surgery. J Cardiovasc Surg 1997; 38: 639-43.

24) Albes JM, Schistek R, Baier R, et al. Intestinal ischemia associated with cardio-pulmonary-bypass surgery: a life threatening complication. J Cardiovasc Surg (Torino) 1991; 32: 527-33.

25) Hanks JB, Curtis SE, Hanks BB, et al. Gastrointestinal complications after cardiopulmonary bypass. Surgery 1982; 92: 394-400.

26) Welling RE, Rath R, Albers JE, et al. Gastrointestinal complications after cardiac surgery. Arch Surg 1986; 121: $1178-80$.

27) Aranha GV, Pickleman J, Pifarre R, et al. The reasons for gastrointestinal consultation after cardiac surgery. Am Surg 1984; 50: 301-4. 